Artículo Aceptado para su pre-publicación / Article Accepted for pre-publication

Título / Title:

Implantes subperiósticos personalizados para la rehabilitación completa del maxilar superior atrófico. Revisión de una serie clínica de 8 casos / Subperiosteal personalised implants for the rehabilitation of the severely deficient edentulous maxilla. Revision of a clinical series of eight cases

\author{
Autores / Authors: \\ Manuel Chamorro Pons, Javier Arias Gallo, Luis Margarit Pérez, Gastón Demaría \\ Martínez, Ángel Cidad Vicario
}

DOI: $\underline{10.20986 / \text { recom.2021.1293/2021 }}$

Instrucciones de citación para el artículo / Citation instructions for the article: Chamorro Pons Manuel, Arias Gallo Javier, Margarit Pérez Luis, Demaría Martínez Gastón, Cidad Vicario Ángel. Implantes subperiósticos personalizados para la rehabilitación completa del maxilar superior atrófico. Revisión de una serie clínica de 8 casos / Subperiosteal personalised implants for the rehabilitation of the severely deficient edentulous maxilla. Revision of a clinical series of eight cases. j.maxilo 2021. doi: 10.20986/recom.2021.1293/2021.

\title{
INSPIRA NETWORK
}

Este es un archivo PDF de un manuscrito inédito que ha sido aceptado para su publicación en la Revista Española de Cirugía Oral y Maxilofacial. Como un servicio a nuestros clientes estamos proporcionando esta primera versión del manuscrito en estado de prepublicación. El manuscrito será sometido a la corrección de estilo final, composición y revisión de la prueba resultante antes de que se publique en su forma final. Tenga en cuenta que durante el proceso de producción se pueden dar errores, lo que podría afectar el contenido final. El copyright y todos los derechos legales que se aplican al artículo pertenecen a la Revista Española de Cirugía Oral y Maxilofacial. 
IMPLANTES SUBPERIÓSTICOS PERSONALIZADOS PARA LA REHABILITACIÓN COMPLETA DEL MAXILAR SUPERIOR ATRÓFICO. REVISIÓN DE UNA SERIE CLÍNICA DE 8 CASOS

\section{SUBPERIOSTEAL PERSONALISED IMPLANTS FOR THE REHABILITATION OF THE SEVERELY DEFICIENT EDENTULOUS MAXILLA. REVISION OF A CLINICAL SERIES OF EIGHT CASES}

Manuel Chamorro Pons ${ }^{1}$, Javier Arias Gallo ${ }^{1}$, Luis Margarit Pérez ${ }^{1}$, Gastón Demaría Martínez ${ }^{1}$ y Ángel Cidad Vicario ${ }^{2}$

${ }^{1}$ Servicio de Cirugía Oral y Maxilofacial y Odontología. Complejo Hospitalario Ruber Juan Bravo. Madrid, España. ${ }^{2}$ Clínica Cidad. Madrid, España

\section{CORRESPONDENCIA:}

Javier Arias Gallo

javierariasgallo@gmail.com

Recibido: 07-06-2021

Aceptado: 04-11-2021

\section{RESUMEN}

Introducción: Los implantes subperiósticos personalizados se presentan como una alternativa en la rehabilitación implantosoportada de pacientes con atrofia ósea grave. Este artículo describe nuestra experiencia en su uso para la rehabilitación completa del maxilar superior.

Pacientes y métodos: Se presenta un estudio retrospectivo de 8 pacientes edéntulos con atrofia ósea grave del maxilar superior, tratados mediante estructuras de titanio personalizadas diseñadas y fabricadas en conjunto con la empresa Avinent Digital Health (Barcelona, España).

Las estructuras se diseñan a partir de un TC maxilomandibular con cortes finos. Como guía de referencia se emplea una prótesis completa acrílica con marcas radiopacas. Las 
estructuras se diseñan en dos o tres piezas para favorecer su inserción y adaptación a los contornos óseos. La cirugía se lleva a cabo bajo anestesia general, e incluye colgajo de bola de Bichat bilateral previo al cierre mucoso. Inmediatamente tras la cirugía se coloca una prótesis fija provisional implantosoportada, que se sustituye por una prótesis fija de cerámica o híbrida a las 8-12 semanas.

Resultados: Todos los pacientes llevan actualmente la prótesis fija final, con un tiempo de seguimiento de 4 a 36 meses. En ningún caso ha sido necesario retirar ni recortar el implante. Todos los pacientes están asintomáticos.

Conclusión: Aunque se trata de un estudio preliminar, los resultados indican que esta técnica debe ser considerada una alternativa de tratamiento en atrofias maxilares graves.

Palabras clave: Implantes subperiósticos, maxilar atrófico, implantes dentales a medida, implantes customizados.

\section{ABSTRACT}

Introduction: Customized subperiosteal implants are presented as an alternative in the implant-supported rehabilitation of patients with severe bone atrophy. This article describes our experience in their use for complete maxillary rehabilitation.

Patients and methods: A retrospective study of 8 edentulous patients with severe maxillary bone atrophy treated with customized titanium frameworks designed and manufactured in conjunction with Avinent Digital Health (Barcelona, Spain) is presented. The frameworks are designed based on a thin-slice maxillomandibular CT scan. A full acrylic prosthesis with radio-opaque markings is used as a reference guide. The structures are designed in two or three pieces to favor their insertion and adaptation to the bone contours. The surgery is performed under general anesthesia, and includes bilateral buccal fat pad flap prior to mucosal closure. Immediately after surgery an implant-supported temporary fixed prosthesis is placed, which is replaced by a fixed ceramic or hybrid prosthesis after 8-12 weeks. 
Results: All patients are currently wearing the final fixed prosthesis, with a follow-up time of 4 to 36 months. In no case has it been necessary to remove or trim the implant. All patients are asymptomatic.

Conclusion: Although this is a preliminary study, the results indicate that this technique should be considered as a treatment alternative in severe maxillary atrophy.

Keywords: Subperiosteal implant, atrophic maxilla, patient-specific implant, customised dental implant.

\section{INTRODUCCIÓN}

Los implantes dentales endoóseos son la técnica de elección para la fijación de prótesis dentales desde hace más de 30 años $^{1}$. Para la colocación y supervivencia a largo plazo de estos implantes, uno de los requisitos más importantes es la existencia de un volumen de hueso con una densidad adecuada para conseguir estabilidad ósea primaria y osteointegración a largo plazo. Cuando el hueso de los maxilares es insuficiente, debe llevarse a cabo procedimientos de injerto óseo, muchas veces complejos, que hacen más impredecible el resultado final, y alargando durante muchos meses el tratamiento. Cawood y Howell ${ }^{2}$ clasificaron las arcadas dentales edéntulas en 6 tipos, dependiendo del grado de atrofia ósea. En las atrofias $\mathrm{V}$ (atrofia completa vertical y horizontal del hueso alveolar) y VI (reabsorción del hueso basal), las opciones terapéuticas sin el empleo de injertos son muy escasas. Los implantes cigomáticos podrían ser una de ellas, pero siguen siendo tratamientos utilizados por un pequeño número de profesionales. Esto es debido a su complejidad técnica, a complicaciones a largo plazo y a los problemas inherentes a las prótesis dentales en estos casos.

Los implantes subperiósticos se propusieron en 1942 por Dahl ${ }^{3}$. Unos años después se presentaron casos clínicos en la literatura, inicialmente para restauraciones unitarias o de pequeños segmentos edéntulos ${ }^{4}$, y posteriormente para rehabilitaciones de arcadas completas $^{5}$. El tratamiento incluía habitualmente un procedimiento quirúrgico inicial de toma de impresión directa sobre el hueso, la fabricación de la estructura subperióstica en el laboratorio de prótesis, y la colocación en una segunda intervención quirúrgica. Incluso se llegaron a publicar algunos casos en los que solo era necesaria una 
intervención ${ }^{6}$. Aun así, la complejidad del tratamiento influyó en la escasa aceptación general de esta técnica en la comunidad médica y dental de mediados del siglo pasado. La revolución digital de los últimos años incluye múltiples avances en distintos campos, como la mejora en la adquisición de datos con los nuevos sistemas de tomografía computarizada y de escáneres intraorales. También el avance de los sistemas de diseño y fabricación por ordenador (CAD-CAM), los nuevos materiales, las nuevas superficies y los nuevos sistemas de fabricación de metal ("direct metal laser sintering"), todo ello permite rescatar el concepto de implantes subperiósticos del siglo pasado para usarlos en pacientes en los que resulta complejo o inviable la colocación de implantes endoóseos habituales ${ }^{7-9}$.

\section{MATERIAL Y MÉTODOS}

Presentamos 8 casos consecutivos de pacientes con atrofia ósea maxilar superior extrema y completa, tipos V y VI de Cawood y Howell, tratados mediante implantes subperiósticos a medida, diseñados en colaboración con una única empresa, Avinent Digital Health ${ }^{\circledR}$ (Barcelona, España). Todos los pacientes han prestado su consentimiento informado respecto a la intervención quirúrgica y a su inclusión en el estudio.

Los criterios de inclusión son los siguientes: edentulismo maxilar completo, atrofia ósea maxilar clases V-VI de Cawood y Howell ${ }^{2}$ (pérdida de proceso alveolar o reabsorción del hueso basal) y ausencia de enfermedades sistémicas que desaconsejaran la colocación de implantes dentales.

El proceso se lleva a cabo de la siguiente manera.

\section{Preparación del caso y diseño de la estructura}

El primer paso es la elaboración de una prótesis provisional, si el paciente no tuviera una previamente. En ella se labran unos pequeños orificios en los cíngulos de los incisivos y caninos, y en las superficies oclusales de premolares y molares, en los que se introducen bolitas metálicas de $1 \mathrm{~mm}$ que se fijan con resina autopolimerizable. 
Con la prótesis puesta en boca, se realiza un TC helicoidal o CBCT con cortes de $1 \mathrm{~mm}$, que la empresa productora del implante recibe directamente en formato DICOM. EI diseño de cada caso se lleva a cabo en coordinación con los ingenieros de la empresa mediante videoconferencia y con las siguientes premisas:

- Emplear la mínima cantidad de material posible. El grosor de la estructura es de $0,8 \mathrm{~mm}$, y está diseñado en forma de tirantes que se adaptan a las zonas de mayor densidad ósea, dejando grandes espacios libres para que la mayor parte de la mucosa y encía descansen directamente sobre el hueso. Se evita, por tanto, cualquier diseño tipo plancha o placa agujereada.

- La estructura abraza el reborde alveolar (si quedara algo de él), y se extiende al hueso basilar, a los arbotantes lateronasales, cigomático-maxilares y el hueso palatino. La estructura se fija mediante tornillos de osteosíntesis de 1,5 $\mathrm{mm}$ de diámetro en las zonas con mayor grosor óseo, tanto en el lado vestibular como en el lado palatino. Se pone especial cuidado en que toda la estructura apoye en el hueso de soporte, sin huecos entre ambos.

- De la estructura descrita emergen unos cilindros que recibirán y fijarán la prótesis. Terminan en una conexión de implantes estándar de hexágono externo. La posición y angulación de los cilindros se hace coincidir con la emergencia ideal para la prótesis dental final.

El número de conexiones para la prótesis es de 6, en posiciones 3-5-7 o 2-4-6.

- La estructura se fabrica en dos o tres piezas: la pieza principal y las extensiones hacia el arbotante cigomático maxilar, que se atornillan con la pieza principal y al hueso. Este diseño desarmado facilita la colocación de la estructura, evitando despegamientos excesivos y desgarros mucosos (Figura 1).

- Junto con la estructura y los tornillos de fijación de titanio recibimos un modelo 3D del maxilar del paciente y una copia de la estructura en poliamida. Esta copia sirve al odontólogo para preparar la prótesis transicional y también se usa como guía en la intervención (Figura 2).

El diseño de la estructura técnicamente se realiza de la siguiente manera: se comienza haciendo la reconstrucción de un modelo 3D virtual del maxilar superior a partir del TC. Para ello se utiliza el software Materialise Mimics ${ }^{\circledR}$. Una vez obtenido este modelo 
virtual de trabajo, se definen las posiciones y orientaciones óptimas de los cilindros emergentes de conexión para la prótesis. El siguiente paso es posicionar la ubicación de los tornillos de osteosíntesis que fijarán la estructura al hueso, eligiendo para ello las zonas de mayor densidad ósea. Con las posiciones de fijación obtenidas, utilizando el software Materialise 3-matic ${ }^{\circledR}$, se dibuja y define la forma de la estructura, así como su grosor. Se obtiene también el tamaño máximo de los tornillos en cada punto (Figura 3). El diseño finalizado se exporta en un fichero con formato.st/ para proceder a su fabricación.

La fabricación se hace en dos pasos. Primero se imprime la estructura de titanio con sus conexiones, ya que por su tamaño y forma no se puede obtener por fresado sino mediante impresión 3D. Las conexiones quedan impresas como cilindros sólidos. Posteriormente estos cilindros de fijación se terminan mediante microfresado, lo que permite obtener un grado óptimo de precisión en la zona de conexión con la futura prótesis. Posteriormente se repasa y pule la superficie. Por último, se limpia y termodesinfecta, quedando lista para esterilizar al autoclave en el quirófano.

\section{Intervención quirúrgica}

El procedimiento se lleva a cabo bajo anestesia general. Se emplea profilaxis antibiótica con amoxicilina/clavulánico $2 \mathrm{~g} / 125 \mathrm{mg}$, e infiltración anestésica local con vasoconstrictor en los surcos labiogingivales y en la encía. Se realiza una incisión crestal con descargas amplias muy posteriores. Es importante realizar la incisión en la encía adherida, dejando una banda de esta tanto en el colgajo vestibular como en el palatino. De esta forma al suturar, los cilindros de fijación quedarán rodeados de encía adherida en toda su circunferencia.

El despegamiento subperióstico debe ser amplio y muy cuidadoso, tanto de la vertiente vestibular como palatina, y debe extenderse a toda la superficie ósea que va a recibir la estructura. La copia de la estructura en poliamida se utiliza para ir comprobando en qué punto el despegamiento subperióstico es suficiente (Figura 4). Finalmente, extraemos la estructura definitiva de titanio de su contenedor estéril y la colocamos en su posición definitiva sobre el hueso maxilar. Esto evita un manejo excesivo de esta, que podría acarrear contaminación, caídas accidentales, etc. 
A continuación, se procede a fijar la estructura con tornillos de osteosíntesis (Figura 5), de grosor y diámetro ya determinados durante la fase de diseño y recogidos en un esquema (Figura 3).

Se obtiene un colgajo bilateral de bola de Bichat para cubrir la parte vestibular de la estructura. Finalmente se lleva a cabo el cierre gingival con sutura reabsorbible de $4 / 0$ (Figura 6).

\section{Prótesis provisional inmediata}

El prostodoncista adapta unos pilares acrílicos a los cilindros de fijación y coloca la prótesis transicional que queda fijada a estos con acrílico autopolimerizable. Los bordes de la prótesis se fresan y pulen. La prótesis provisional, queda fija durante 1,5-2 meses (Figura 7).

El paciente es dado de alta el mismo día, con analgesia pautada, tratamiento antibiótico oral (amoxicilina/clavulánico 500/125 mg) durante los primeros 7 días y enjuagues con colutorio de clorhexidina 5-6 veces al día durante 2 semanas.

\section{Prótesis definitiva}

Tras 1,5-2 meses, una vez la encía está completamente cicatrizada, se efectúa la toma de impresiones para el diseño y fabricación de la prótesis definitiva. En 6 casos se ha realizado prótesis fija en metal porcelana. La estructura metálica de la prótesis se diseña en Cad-Cam y se fresa en cromo-cobalto. Exteriormente es cerámica con revestimiento multicromático de alta fusión trabajado con técnica de pincel (Figura 8). En 2 casos se ha realizado prótesis híbrida de resina (Figura 9).

\section{RESULTADOS}

Los 8 casos que presentamos han completado todas las fases de tratamiento y se encuentran rehabilitados con la prótesis definitiva (Tabla I). El periodo de evolución va desde los 3 años en el primer caso a los 4 meses para el caso último (n. 8). 
Todos ellos se han intervenido bajo anestesia general en régimen de hospital de día. Solo 1 caso ( $n$. o 6) pernoctó en el hospital debido a que se trataba de un paciente tratado crónicamente con anticoagulación oral y las indicaciones preanestésicas así lo recomendaban.

El tiempo quirúrgico empleado para la colocación de la estructura y cierre mucoso ha oscilado desde los 65 minutos a los 95, siendo la media 80 minutos. Para la colocación de la prótesis provisional, el prostodoncista ha empleado una media de 50 minutos. La precisión de la estructura se consideró en todos los casos impecable, sin cabeceos ni huecos entre el hueso y el implante. La estabilidad de los tornillos de osteosíntesis fue siempre adecuada. En algún caso aislado, hemos sustituido el tornillo previamente asignado por uno de rescate, más grueso, al no producirse una fijación inicial óptima. Cuando el hueso receptor del tornillo de osteosíntesis tenía menos de $4 \mathrm{~mm}$ de espesor se infrapreparaba el lecho óseo buscando una entrada y progresión del tornillo con cierta resistencia.

Una vez dados de alta, los pacientes fueron citados para revisión a los 7, 15, 30 y 45 días. La evolución de los pacientes ha sido muy buena en todos los casos. No hemos tenido ninguna complicación reseñable en el corto plazo relacionada con infección o dehiscencia de suturas. Después de la última revisión (45 días postoperatorios) enviamos al paciente al prostodoncista para comenzar con el diseño y toma de medidas para la elaboración de la prótesis definitiva. Ha habido variaciones importantes en el tiempo empleado en colocar la prótesis definitiva, que ha oscilado entre los 65 y 90 días postoperatorios $(57,5)$, debido a repeticiones de la toma de medidas, cambios de citas, etc.

Ya con la prótesis definitiva, los pacientes son revisados periódicamente cada 4 meses. En estas revisiones se realiza desmontado de la prótesis si se precisa, revisión de los cilindros de fijación, limpieza, etc. A medio y largo plazo no hemos observado ninguna complicación reseñable. No hemos tenido infecciones, ni exposición de la estructura, ni ha sido necesario extraer tornillos de osteosíntesis. En uno de los casos (n. ㅇ 3) tuvimos un episodio de inflamación gingival y dolor local que se debió a un roce traumático de la prótesis sobre la encía que originó una ulceración superficial e inflamación reactiva local. La complicación se solucionó con el desmontaje y retoque de la prótesis y utilización de gel de clorhexidina en la zona. 


\section{DISCUSIÓN}

La atrofia ósea de los maxilares edéntulos es el principal inconveniente para la colocación de implantes endoóseos.

La clasificación más empleada de la atrofia ósea según su intensidad es la de Cawood y Howell ${ }^{2}$. En ella se distinguen las clases de atrofia ósea tipo $V$, con reborde óseo con inadecuada anchura y altura; y tipo VI, en los que además encontramos depresiones y defectos estructurales.

El tratamiento de los casos de atrofia grave (tipo $\mathrm{V}$ y VI) es ciertamente complicado. Las dimensiones del hueso remanente son tan escasas que técnicas más habituales, como el empleo de injertos óseos, tienen un resultado mucho más impredecible, porque el lecho receptor es inadecuado para soportar, fijar y dar viabilidad a los injertos. En el trabajo que presentamos, todos los pacientes $(n=8)$ presentaban atrofias extremas en toda la extensión del maxilar, por lo que la indicación de una estructura subperióstica está justificada.

La única alternativa posible habría sido plantear un cuádruple implante cigomático. Como es sabido, en los casos en los que hay suficiente volumen de hueso en la zona anterior del maxilar, la combinación implantes convencionales anteriores con un implante cigomático a cada lado no resulta un proceso excesivamente complejo. Sin embargo, si no hay suficiente hueso disponible en la premaxila para los implantes estándar, se deben colocar dos implantes cigomáticos a cada lado ${ }^{10}$, lo que complica bastante la cirugía desde el punto de vista técnico. La emergencia de los implantes cigomáticos es con mucha frecuencia demasiado palatina, lo que complica la estabilidad, higiene y fonética de la prótesis definitiva. Por otro lado, se acepta que la patología crónica rinosinusal previa aumenta las complicaciones de los implantes cigomáticos, incluso con la técnica extrasinusal ${ }^{10}$. Sin embargo, el diseño personalizado de los implantes subperiósticos hace que se puedan mantener completamente alejados del seno maxilar, lo que hace suponer que las complicaciones sinusales no sean relevantes. Para las cirugías extensas en la cavidad oral, incluyendo los implantes subperiósticos de una arcada completa, nosotros proponemos la utilización de anestesia general en régimen de hospital de día, tanto por comodidad del paciente como por su seguridad. 
El despegamiento subperióstico es muy extenso. Puede haber riesgo de aspiración de sangre, secreciones, instrumentos o tornillos de osteosíntesis cuando la cirugía se realiza con anestesia local. El riesgo es incluso mayor en pacientes sedados. Aunque seguramente un cirujano maxilofacial con experiencia pueda realizar esta intervención con éxito bajo anestesia local y sedación, nosotros creemos que el ligero aumento del coste de la intervención que supone el uso de un quirófano y la intervención de un anestesista está plenamente justificado.

En los casos que presentamos en este estudio, además de la atrofia extrema de la cresta maxilar, hemos podido observar defectos estructurales importantes debidos a cirugías previas, pérdida de implantes convencionales, etc. En el caso n.ำ 1 existía un defecto óseo completo en la vertiente palatina derecha sin solución de continuidad con la fosa nasal, debido a cirugías previas por infecciones de repetición en los molares de ese lado (Figura 2).

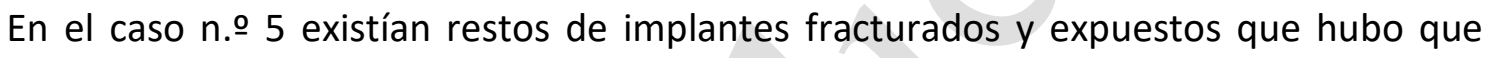
retirar previamente. $Y$ en el caso $n .07$ se retiraron dos implantes de forma simultánea a la colocación de la estructura.

Se asume generalmente que los movimientos de la estructura sobre el hueso son perjudiciales para su supervivencia a largo plazo. El diseño computarizado de la estructura a partir de un TC con cortes finos permite una adaptación pasiva y sin espacios muertos entre el hueso y el metal. Los tornillos de osteosíntesis dan una fijación rígida sobre todo los primeros meses, antes de que el periostio contribuya a la fijación de la estructura. Los primeros implantes subperiósticos históricos, con todos sus fallos de diseño e imprecisiones, no utilizaban tornillos de osteosíntesis ${ }^{4,5}$, o tan solo dos tornillos en una rehabilitación mandibular ${ }^{11}$, y aun así podían tener una fijación razonablemente estable durante años. No hay estudios modernos sobre la necesidad o no de tornillos de osteosíntesis concretamente para esta indicación. No obstante, parece razonable suponer que la inmovilización con tornillos de osteosíntesis de una estructura que además apoya pasivamente sobre el hueso será una maniobra quirúrgica favorable a corto y a largo plazo. Nosotros proponemos de modo empírico al menos 1416 tornillos en maxilar, que es el número de tornillos habitual a cada lado de la osteotomía de Lefort I en cirugía ortognática (Figura 5). Los movimientos verticales de compresión son muy bien tolerados, dado que toda la estructura se apoya en el hueso. 
Quizá el movimiento más desfavorable es el de tracción, lo cual es un motivo mayor para evitar prótesis cementadas o removibles, y aconsejar que el paciente evite comer alimentos adherentes, como ciertos dulces.

Proponemos además un diseño con un cuerpo principal de la estructura y extensiones laterales independientes que se atornillan simultáneamente a la estructura principal y al hueso malar (Figura 1). De este modo se maximiza la fijación ósea reduciendo a la vez la cantidad de despegamiento subperióstico requerido. Así resulta más sencilla la entrada de la estructura bajo el colgajo realizado. En los 4 últimos casos hemos realizado un diseño en 2 piezas, haciendo desmontable solo una de las prolongaciones laterales de la estructura. Observamos que este despiece unilateral es suficiente para evitar despegamientos excesivos, y la entrada de la estructura se sigue realizando con facilidad.

En nuestra serie, los cilindros de fijación, que conectan la estructura principal con la prótesis, se sitúan en los puntos ideales desde el punto de vista protésico. La emergencia y angulación de estos pilares de conexión se diseñan individualmente en cada caso. Se tiene en cuenta que la existencia y el mantenimiento de una encía queratinizada alrededor de los pilares es un requisito fundamental de éxito a largo plazo (Figura 8). El número de conexiones protésicas para un maxilar superior es de seis. Consideramos que de este modo la carga masticatoria de la estructura se reparte uniformemente.

En el caso de que algún cilindro de conexión presentara algún problema inflamatorio en el futuro, debe ser posible desconectarlo y extraerlo de un modo sencillo, para no comprometer la función a largo plazo de la prótesis. Mommaerts ${ }^{9}$ insiste mucho en ese aspecto del diseño. Nuestro diseño es ligero. Si fuera necesario, las conexiones entre la estructura principal y la parte en contacto con la prótesis dental pueden cortarse fácilmente con una fresa de diamante bajo anestesia local. Disponer de seis conexiones protésicas permite que la supervivencia de la estructura a largo plazo no se vea comprometida si ello ocurre.

En los 5 primeros casos que realizamos, la terminación de los cilindros de fijación era en forma de hexágono externo compatible. Esta era la terminación que nos permitía tener un cilindro de emergencia lo más corto posible, más corto incluso que si se usaba una conexión interna. En los últimos casos $(6,7$ y 8 ) hemos comenzado a utilizar una terminación del cilindro tipo Multiunit ${ }^{\circledR}$. Con este sistema hemos conseguido acortar 
significativamente la longitud del cilindro que conecta la estructura subperióstica con la prótesis (Figura 10). Esto es muy importante en los casos de encía muy fina, o en los que la dimensión vertical sea algo limitada, como puede ocurrir si existen dientes sobrerupcionados en la arcada contralateral que reduzcan la altura prótesica disponible. Respecto al tipo de prótesis dental, en la aún escasa literatura sobre los implantes subperiósticos se proponen diferentes tipos. Gellrich y cols. ${ }^{3}$ proponen en algunos casos prótesis removibles con conexiones de bola y barra. Sin embargo, desde el punto de vista biomecánico, parece aconsejable que la prótesis sea fija. De esta manera se evita la tracción sobre la estructura, que es el movimiento más desfavorable para la interfase con el hueso, como ya hemos comentado. Hay autores que proponen prótesis fija cementada, con conexiones cónicas ${ }^{12}$. No obstante, la tendencia más actual, que nosotros hemos seguido, es que la emergencia del cilindro de conexión imite un implante convencional, lo que permite hacer estructuras atornilladas. De esta manera pueden revisarse más fácilmente, y desde el punto de vista del odontólogo el tratamiento y las revisiones resulta mucho más familiar. Además, la emergencia de los cilindros de fijación y la posición de la prótesis en el espacio se tienen en consideración durante el diseño de la estructura (Figura 3). No existen, por tanto, problemas de emergencia inadecuada de las conexiones protésicas que obliguen al uso de aditamentos rectificadores de la angulación ni al cementado de la prótesis.

En 6 de los 8 casos hemos utilizado prótesis fija atornillada de metal porcelana. La encía protésica también ha sido fabricada en porcelana con revestimiento multicromático, para maximizar el resultado estético (Figura 8). En 2 casos (casos n.o 4 y 8) se realizó prótesis híbrida de resina (Figura 9). El motivo fue la necesidad de mejorar el gran hundimiento de labios y mejillas que estos pacientes presentaban, lo que requería un grosor importante del material protésico. Estos dos casos así tratados han coincidido con los pacientes de mayor edad (81 y 82 años, respectivamente).

En esta serie no hay recogidas complicaciones graves, ni quirúrgicas, ni de la estructura ni de las prótesis. Esta baja incidencia de complicaciones está en línea con las de otros autores $^{8,12}$ Ello puede ser debido a la atención en la pasividad de las estructuras en el hueso, la preservación del máximo de encía queratinizada y el uso de los colgajos de bola de Bichat para cubrir la parte vestibular de la estructura. Por otro lado, reconocemos que el corto tiempo de evolución puede ser un factor importante para 
tener en cuenta. Otros autores reportan complicaciones, principalmente osteolisis alrededor de la estructura y sus tornillos, y exposiciones parciales de la estructura ${ }^{13}$. Sin duda, son necesarios estudios a largo plazo para determinar la supervivencia a largo plazo de las estructuras personalizadas de este tipo.

\section{CONCLUSIONES}

La rehabilitación con implantes subperiósticos en casos de atrofia maxilar grave logra que el paciente tenga una prótesis fija completamente funcional en pocos meses, con una morbilidad aceptable, y con un manejo odontológico similar a las prótesis sobre implantes convencionales. Este es un estudio de una serie limitada de pacientes, con un tiempo de seguimiento corto. Son necesarios más estudios para valorar las indicaciones de esta técnica.

La presente investigación no ha recibido ayudas específicas provenientes de agencias del sector público, sector comercial o entidades sin ánimo de lucro.

\section{BIBLIOGRAFÍA}

1. Albrektsson T, Dahl E, Enbom L, Engevall S, Engquist B, Eriksson AR, et al. Osseointegrated Oral Implants. J Periodontol. 1988;59(5):287-96. DOI: 10.1902/jop.1988.59.5.287.

2 Cawood JI, Howell RA. A classification of the edentulous jaws. Int J Oral Maxillofac Surg. 1988;17(4):232-6. DOI: 10.1016/S0901-5027(88)80047-X.

3. Gellrich NC, Zimmerer RM, Spalthoff S, Jehn P, Pott PC, Rana M, et al. A customised digitally engineered solution for fixed dental rehabilitation in severe bone deficiency: A new innovative line extension in implant dentistry. Journal of CranioMaxillofacial Surgery. 2017;45(10):1632-8. DOI: 10.1016/j.jcms.2017.07.022.

4 Weinberg BD. Subperiosteal Implantation of a Vitallium (Cobalt-Chromium Alloy) Artificial Abutment. J Am Dent Assoc. 1950;40(5):549-62. DOI: 10.14219/jada.archive.1950.0080. 
5. Bodine RL. Prosthodontic essentials and an evaluation of the mandibular subperiosteal implant denture. J Am Dent Assoc. 1955;51(6):654-64. DOI: 10.14219/jada.archive.1955.0260.

6. Weber SP, Cranin AN. The one-phase subperiosteal implant. J Am Dent Assoc. 1970;81(3):698-700. DOI: 10.14219/jada.archive.1970.0303.

7. Gellrich NC, Rahlf B, Zimmerer R, Pott PC, Rana M. A new concept for implantborne dental rehabilitation; how to overcome the biological weak-spot of conventional dental implants? Head and Face Medicine. 2017;13(1):17. DOI: 10.1186/s13005-017-0151-3.

8. Mangano C, Bianchi A, Mangano FG, Dana J, Colombo M, Solop I, et al. Custommade 3D printed subperiosteal titanium implants for the prosthetic restoration of the atrophic posterior mandible of elderly patients: a case series. 3D Print Med. 2020;6(1):1. DOI: 10.1186/s41205-019-0055-x.

9. Mommaerts MY. Evolutionary steps in the design and biofunctionalization of the additively manufactured sub-periosteal jaw implant 'AMSJI' for the maxilla. Int J Oral Maxillofac Surg. 2019;48(1):108-14. DOI: 10.1016/j.ijom.2018.08.001.

10. Maló P, de Araújo Nobre M, Lopes A, Ferro A, Moss S. Extramaxillary Surgical Technique: Clinical Outcome of 352 Patients Rehabilitated with 747 Zygomatic Implants with a Follow-Up between 6 Months and 7 Years. Clin Implant Dent Relat Res. 2015;17(Suppl1):e153-62. DOI: 10.1111/cid.12147.

11. Weber SP. Complete bilateral subperiosteal implants for partially edentulous mandibles. J Prosthet Dent. 1968;20(3):239-47. DOI: 10.1016/00223913(68)90120-0.

12 Cerea M, Dolcini GA. Custom-Made Direct Metal Laser Sintering Titanium Subperiosteal Implants: A Retrospective Clinical Study on 70 Patients. BioMed Res Int. 2018;2018:5420391. DOI: 10.1155/2018/5420391.

13. Nguyen TM, Caruhel JB, Khonsari RH. A subperiosteal maxillary implant causing severe osteolysis. J Stomatol Oral Maxillofac Surg. 2018;119(6):523-5. DOI: 10.1016/j.jormas.2018.06.007. 
Figura 1. Diseño de la estructura subperióstica con los cilindros de conexión protésica con terminación en hexágono externo. Visión vestibular y palatina. Prolongación lateral izquierda desmontable para facilitar la introducción de la estructura.

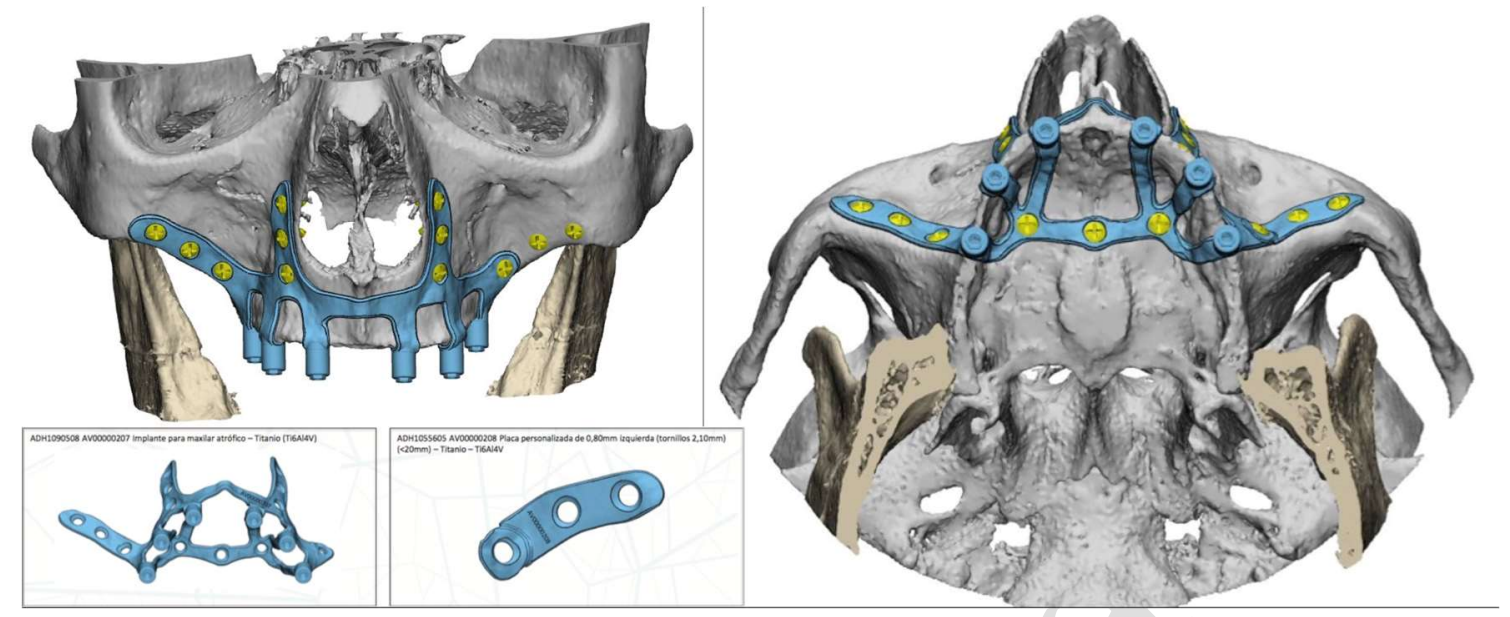


Figura 2. Copia de la estructura en poliamida y su encaje en el modelo 3D del maxilar. Nótese el defecto óseo en palatino que existía en este caso.

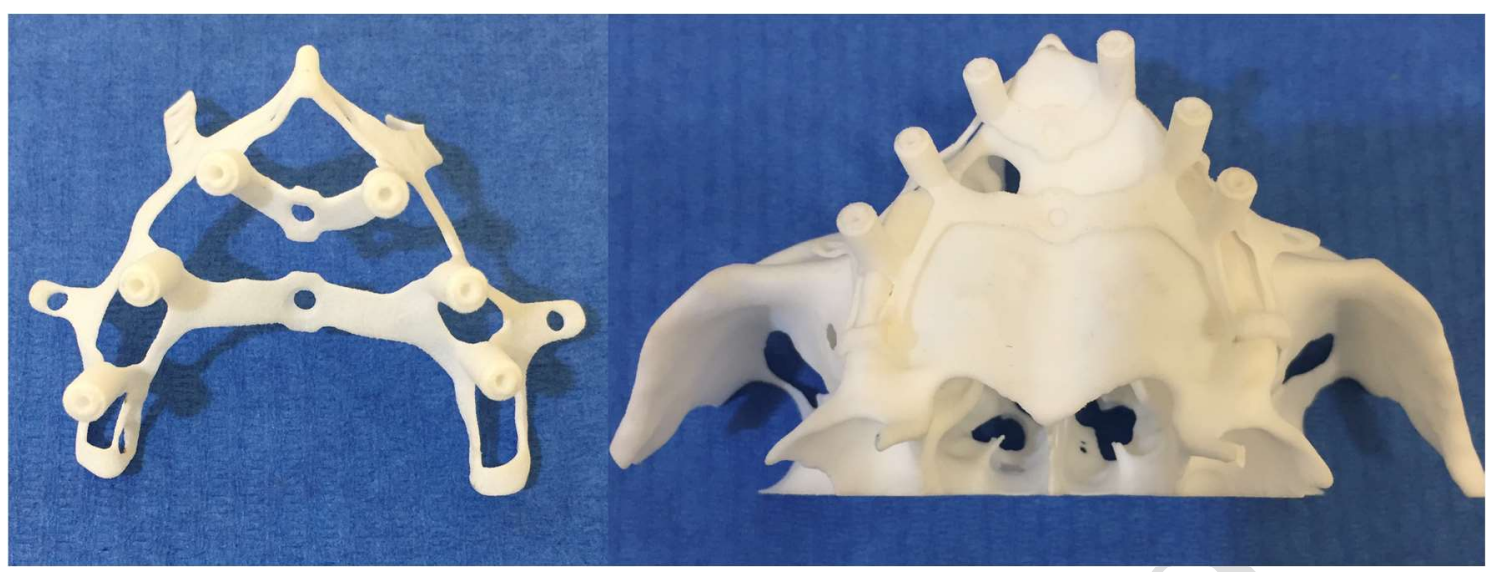


Figura 3. Diseño de la posición y emergencia óptima de los cilindros de fijación para la prótesis. Orientación espacial de la prótesis y esquema de las longitudes de tornillo recomendadas.
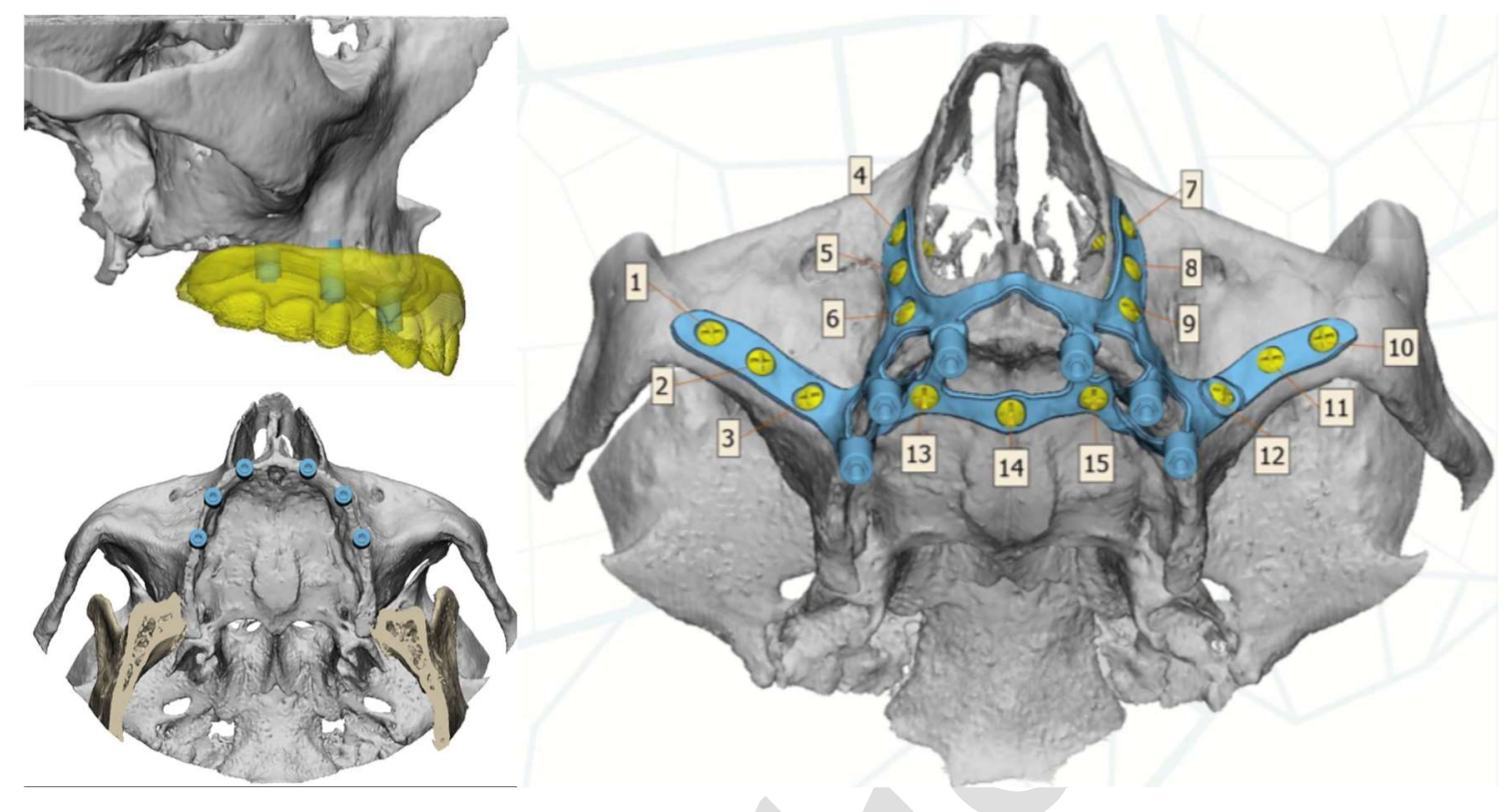
Figura 4. Colocación de prueba de la copia de la estructura en poliamida sobre el hueso. Facilita no tocar la estructura definitiva hasta que se comprueba que el despegamiento es adecuado.

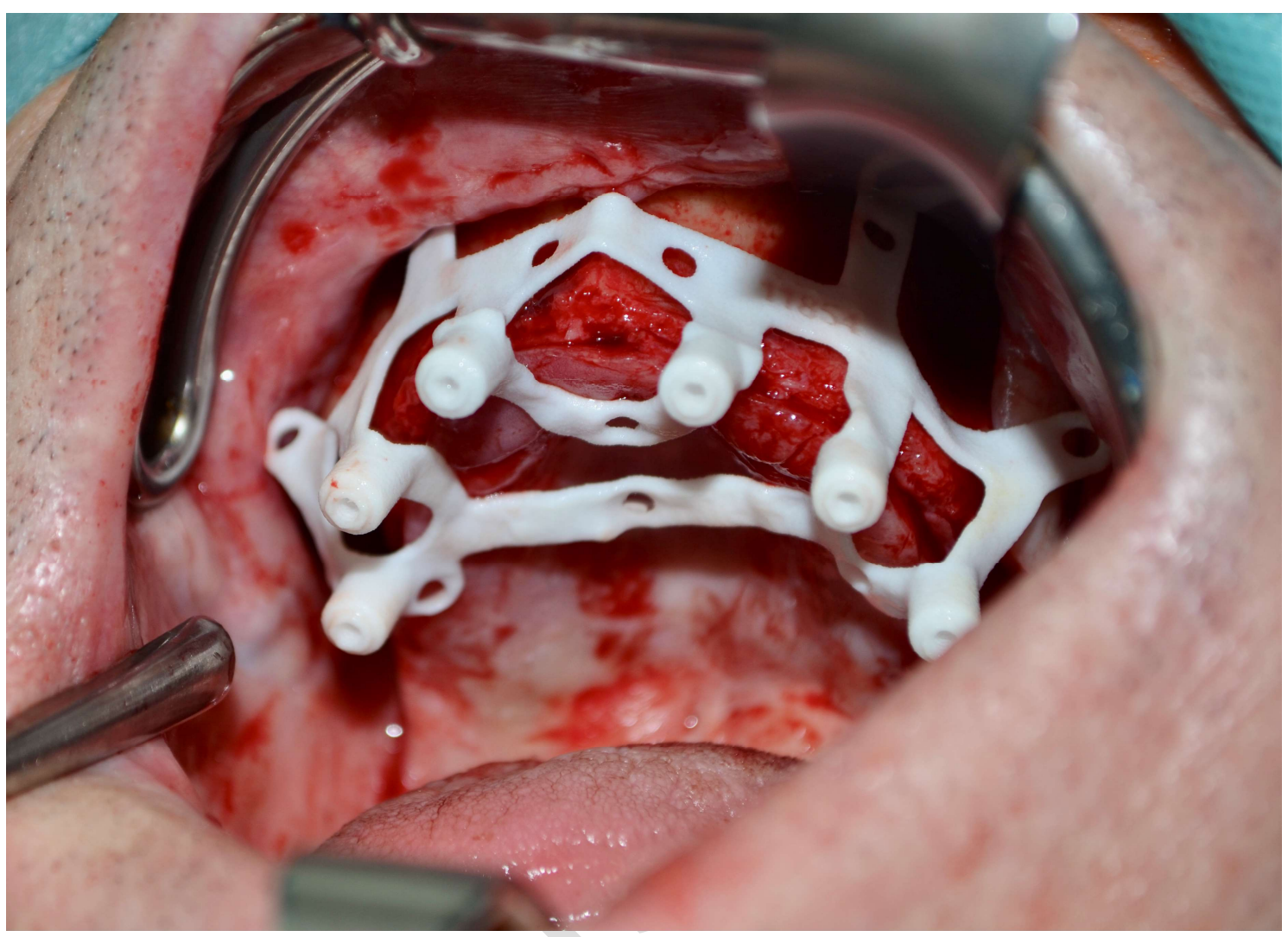


Figura 5. Estructura definitiva de titanio colocada sobre el hueso maxilar en el proceso de fijación, con la parte desmontable de color más azulado.

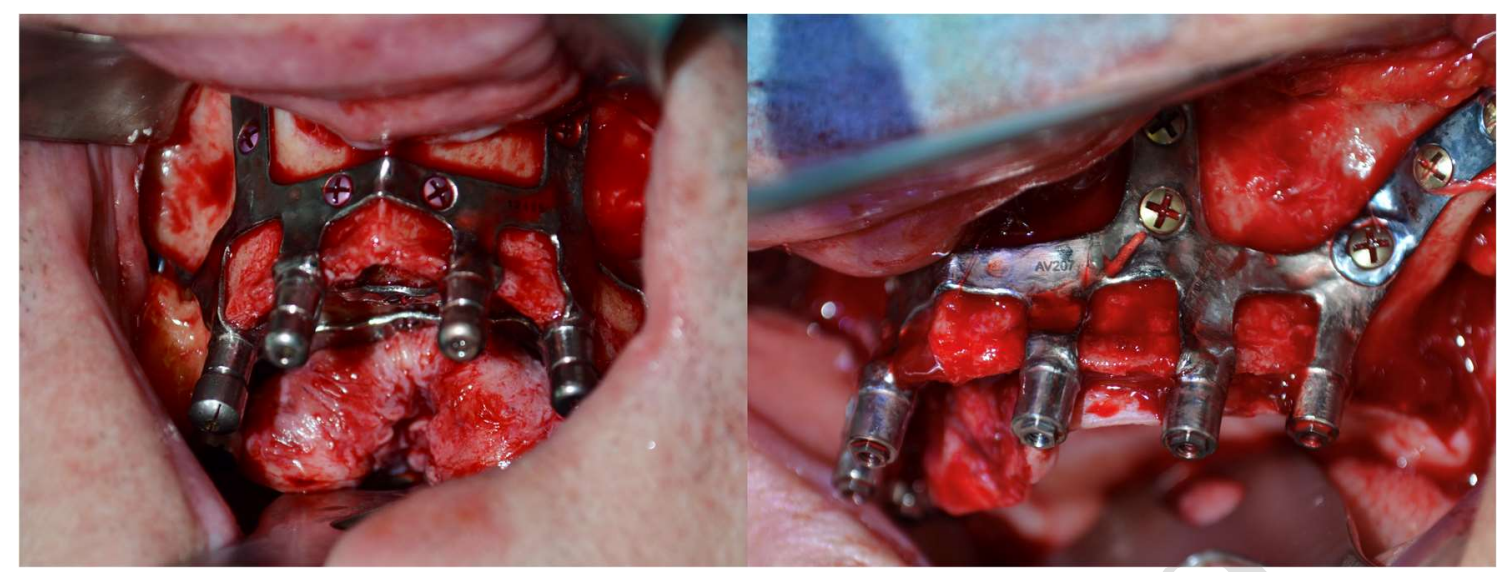


Figura 6. Detalle del cierre de los colgajos interponiendo la bola de Bichat para cubrir el aspecto vestibular de la estructura. Cierre definitivo.

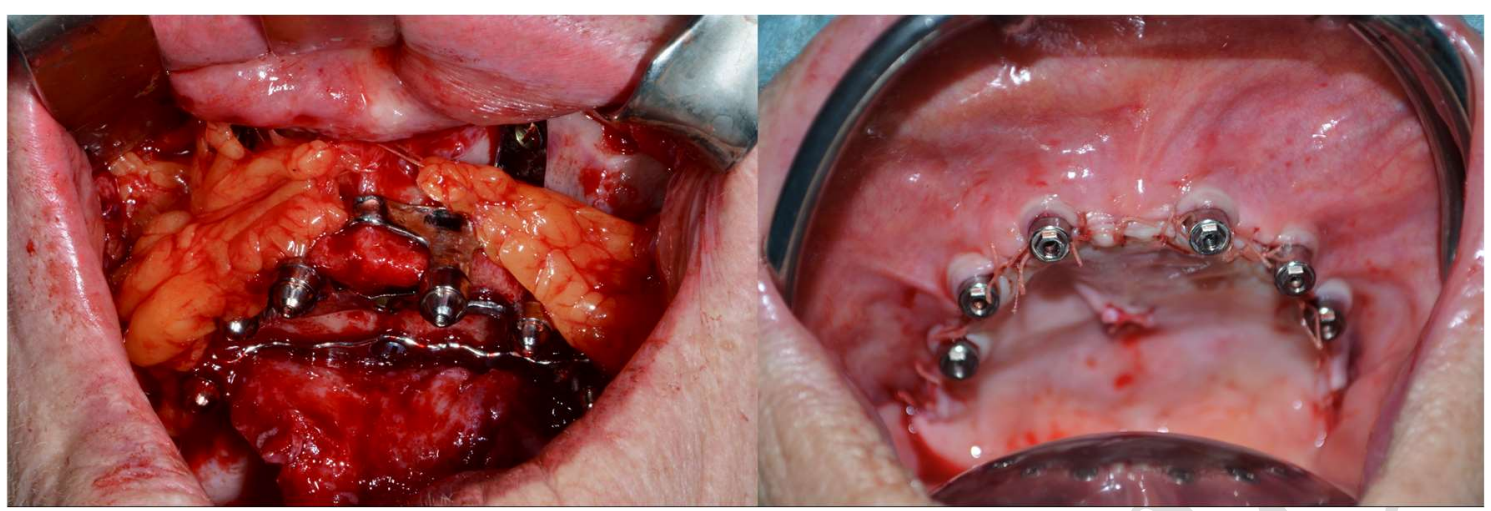


Figura 7. Colocación de la prótesis provisional que va atornillada mediante cilindros de fibra.

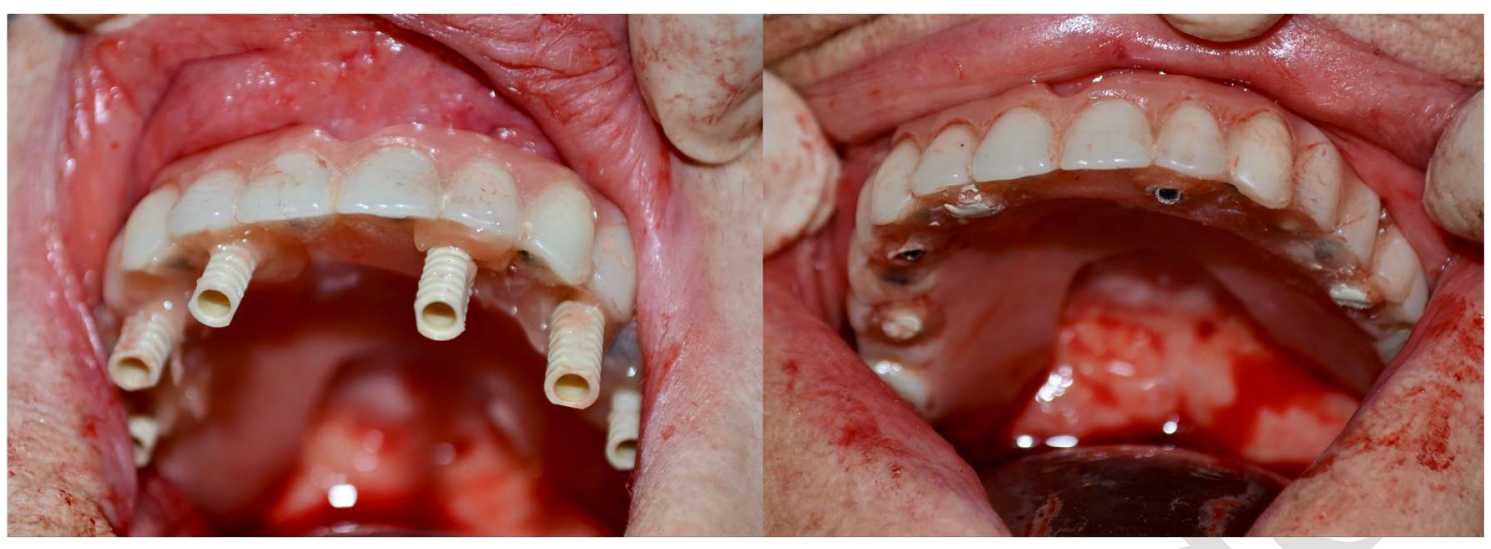


Figura 8. Toma de medidas para la elaboración de la prótesis definitiva. Prótesis definitiva de metal porcelana con recubrimiento de cerámica multicromático.

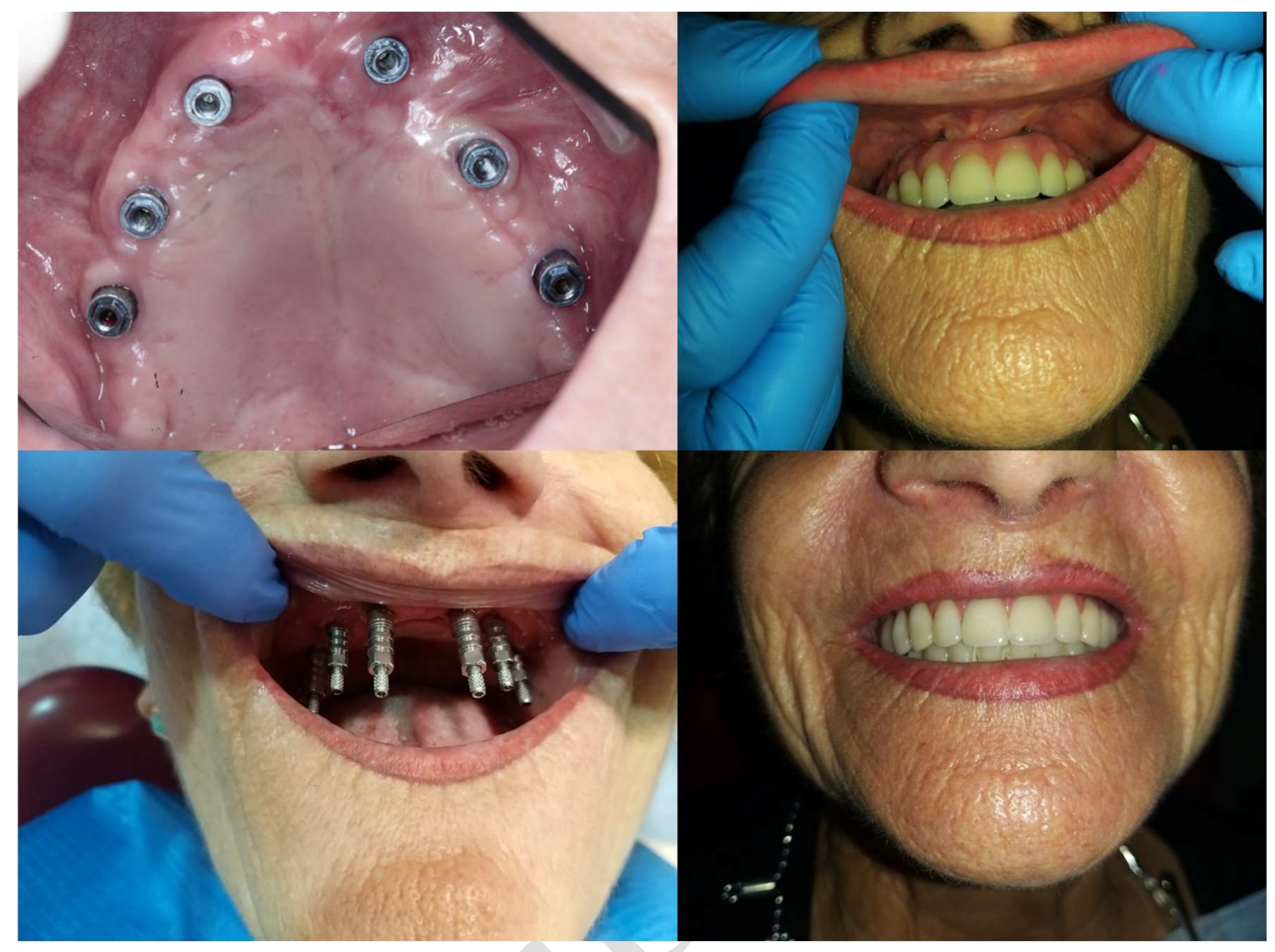


Figura 9. Conexión protésica en un caso de prótesis de resina.

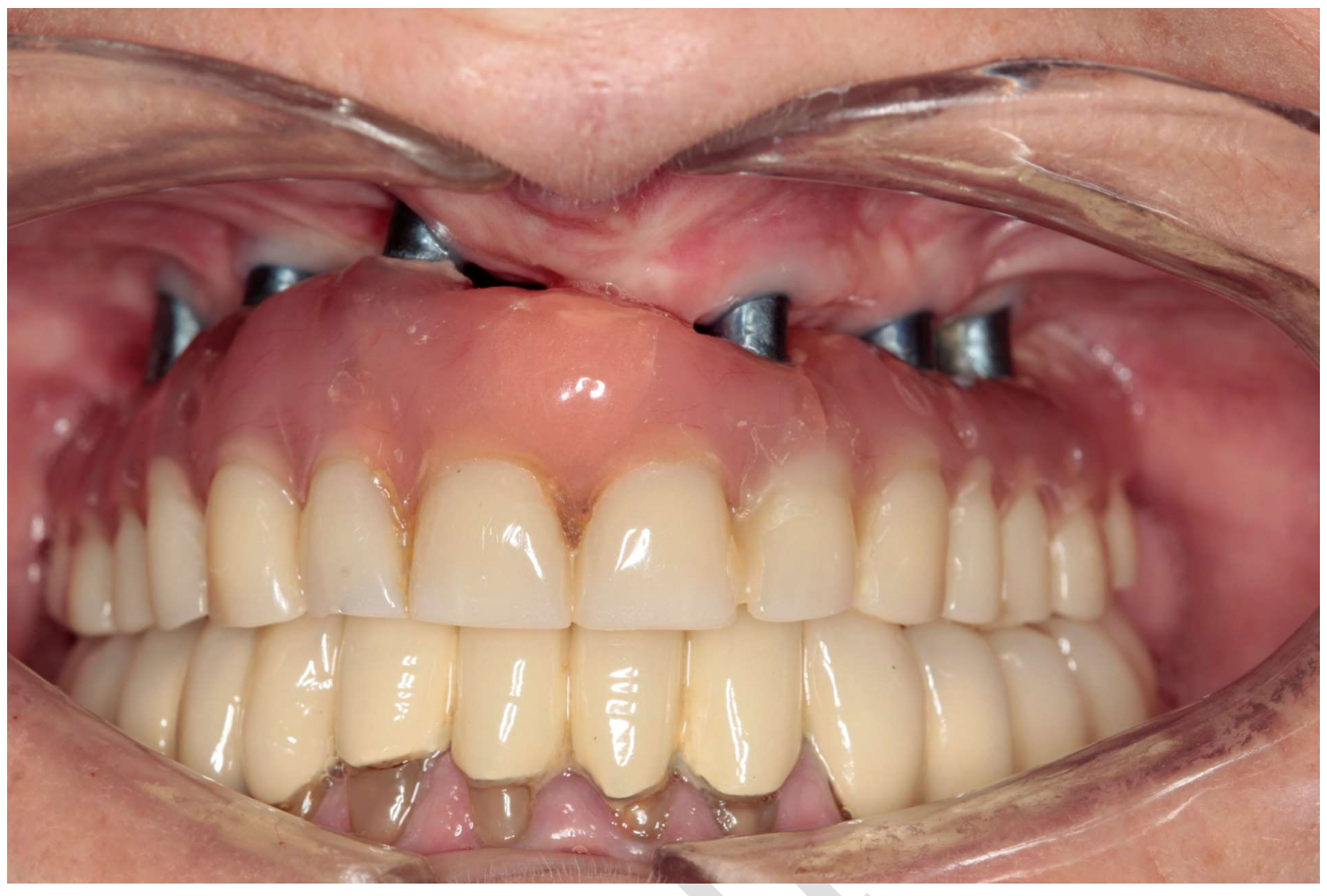


Tabla I. Demografía y tratamiento protésico final.

\begin{tabular}{|c|c|c|c|c|}
\hline Pacientes y sexo & Edad & Tipo de prótesis & $\begin{array}{l}\text { Tiempo } \\
\text { (meses) }\end{array}$ & Tipo de conexión \\
\hline n.․ 1. Mujer & 65 & Metal porcelana & 36 & Hexágono externo \\
\hline n. 2. Mujer & 75 & Metal porcelana & 30 & Hexágono externo \\
\hline n. ㅇ 3. Hombre & 78 & Metal porcelana & 24 & Hexágono externo \\
\hline n. 94 . Hombre & 81 & Híbrida de resina & 18 & Hexágono externo \\
\hline n. o 5. Mujer & 71 & Metal porcelana & 14 & Hexágono externo \\
\hline n. o 6. Mujer & 67 & Metal porcelana & 12 & Multiunit \\
\hline n. ㅇ 7. Mujer & 59 & Metal porcelana & 9 & Multiunit \\
\hline n. o 8. Mujer & 82 & Híbrida de resina & 4 & Multiunit \\
\hline
\end{tabular}


Figura 10. Terminación tipo Multiunit ${ }^{\circledR}$, utilizada en los últimos casos. Permite acortar significativamente la longitud de los cilindros de fijación respecto a la terminación de la Figura 1.

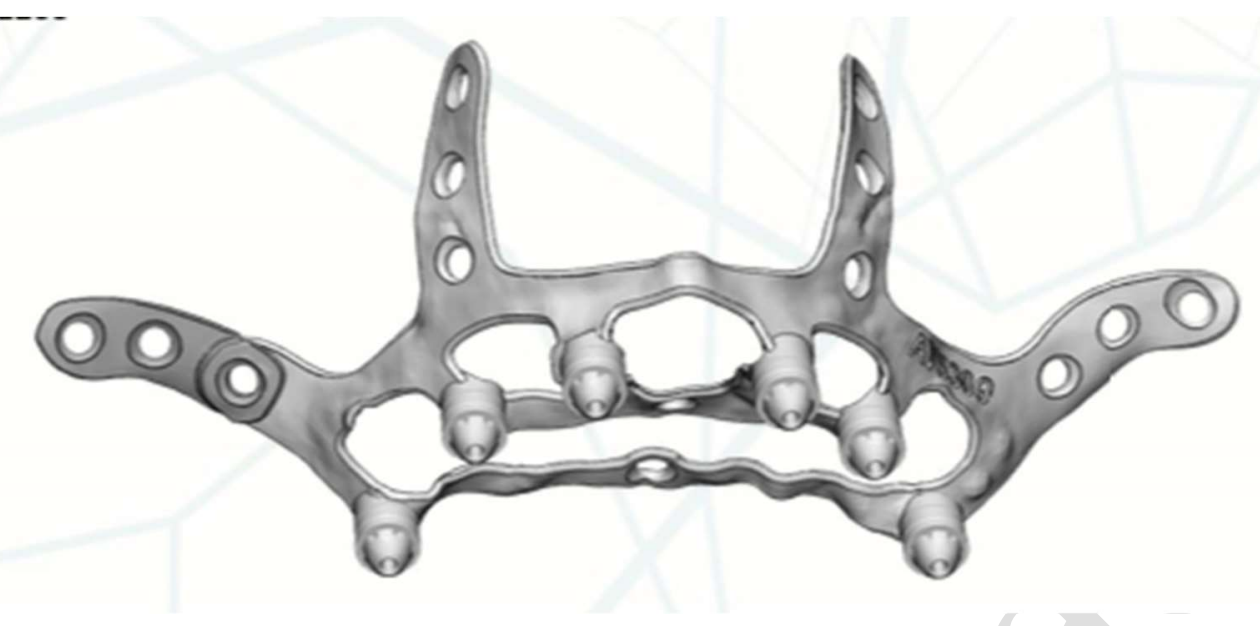

